\title{
Pulmonary Valve Disorder
}

National Cancer Institute

\section{Source}

National Cancer Institute. Pulmonary Valve Disorder. NCI Thesaurus. Code C78579.

A heart disorder characterized by a defect in pulmonary valve structure or function. 